\title{
Seminar Users in the Arabic Twitter Sphere
}

\author{
Kareem Darwish ${ }^{1}$, Dimitar Alexandrov ${ }^{2}$, Preslav Nakov ${ }^{1}$, and Yelena Mejova ${ }^{1}$ \\ 1 Qatar Computing Research Institute, HBKU, Doha, Qatar \\ \{KDarwish,PNakov, YMejova\} @hbku.edu.qa, \\ 2 Sofia University, Bulgaria \\ Dimityr.Alexandrov@gmail.com
}

\begin{abstract}
We introduce the notion of "seminar users", who are social media users engaged in propaganda in support of a political entity. We develop a framework that can identify such users with $84.4 \%$ precision and $76.1 \%$ recall. While our dataset is from the Arab region, omitting language-specific features has only a minor impact on classification performance, and thus, our approach could work for detecting seminar users in other parts of the world and in other languages. We further explored a controversial political topic to observe the prevalence and potential potency of such users. In our case study, we found that $25 \%$ of the users engaged in the topic are in fact seminar users and their tweets make nearly a third of the on-topic tweets. Moreover, they are often successful in affecting mainstream discourse with coordinated hashtag campaigns.
\end{abstract}

Keywords: seminar users, astroturfing, politics, propaganda, malicious users, social media, Twitter, social bots.

\section{Introduction}

What is the connection between a fishing exhibition in Abu Dhabi and ISIS rhetoric? We encountered this puzzle while analyzing ISIS supporters and opponents on Twitter in 2014 [34], finding, to our surprise, the hashtag \#AbuDhabiFishingExhibition ${ }^{3}$ to be one of the most discriminating features. Tracing the accounts that used this hashtag, we found that they had an abnormally high overlap between the tweets they retweeted and the hashtags they used, suggesting they were colluding in some way. However, these accounts did not seem to be political bots [19], which display "interests" in multiple subjects, such as the above-mentioned ones. Neither were they trolls. While their political messages were spirited, they were not provoking others into conflict [24]. They were not selling products or phishing with fake URLs, as spammers would do [5]. However, they were consistent in supporting and promoting the actions of a particular political entity. The closest notion of this behavior in the literature is astroturfing, in which users try to give the impression that a grassroots movement is taking place [46]. Unlike most astroturfing efforts, these users did not mask their identity, and had a persistent political stance over months or years.

\footnotetext{
${ }^{3}$ We translate all tweets and hashtags from Arabic to English to ease readability.
} 
In order to describe users who appear to be real people with persistent political orientation, yet who engage in a coordinated political speech, we use a term originally referring to callers to radio stations who espouse strong political sentiment, namely a "seminar caller." ${ }^{4}$ The term signifies a specific type of person, who receives instructions in a "seminar" on how to deliver talking points on a live talk show effectively, as if sitting in a "seminar" instructing them. Similarly, Twitter seminar users typically attempt to appear like normal individuals, while using specific talking points to promote specific agendas, to manipulate public opinion, and to give the impression of grassroots support. The operating definition that we use for seminar users in this work is the following: "A politically oriented account acting alone or in a group that is dedicated to the consistent support of a specific entity (government, political party, etc.) or its agenda; the account must not be an official account of the entity." A seminar user could be a paid employee of an entity that (s)he actively promotes and could be part of a group of such employees. Incidentally, given our interest in this work is the Arabic Twitter sphere, the term seminar user is closely related to the coined Arabic term for such users namely لجان إلكترونية (lijan electroniyya), which literally translates as "electronic committees".

Increasingly in the Arab world, seminar users are being employed by governments and opposition parties as a propaganda tool to promote specific agendas, to influence public opinion, or to push policies or future directions. For example, on April 13, 2016, General Sisi of Egypt said he could sway social media agenda using electronic brigades. ${ }^{5}$ The activities of such organized users are potentially harmful in a number of ways. From a data processing perspective, they pollute social media streams, and skew measurements of public opinion. From a political and social perspective, they may give the impression of grassroots support for questionable or illegal activities such as physically harming opponents. Here are some examples of such activity:

- Pro-Sisi seminar users promoted the hashtag \#HoldingOnToSisi in the days leading up to the fifth anniversary of the 2011 Egyptian uprising that overthrew Hosny Mubarak. A sample tweet in this campaign states (translation): "\#HoldingOnToSisi \#IAmAnElectronicCommitteeForSisi we are proud electronic committees for our country, our president, our military, and our police."

- Anti-Sisi seminar users promoted the hashtag \#ThePeopleTellSisiWeStronglyReject(you) between December 13-16, 2015. A sample tweet states: "demonstrate and tell injustice to go away, demonstrate and tell Sisi we strongly reject (you) \#ThePeopleTellSisiWeStronglyReject."

Although the phenomenon of seminar users is similar to other phenomena in the field of online political speech, we find no existing definitions that capture this notion in a satisfactory way: these users are not trolls, neither are they astroturfers or bots. In fact, below we will show that state-of-the-art bot detection tools fail to discern these accounts.

\footnotetext{
4 http://en.wikipedia.org/wiki/Seminar_caller

${ }^{5}$ http://albedaiah.com/news/2016/04/13/111001 (in Arabic)
} 
Thus, the contributions of this work are as follows:

- We present a method for the automatic detection of seminar users that engage in political discourse in the Arab world. We manually label users who are engaged in political discourse related to Egypt, United Arab Emirates, Saudi Arabia, and Yemen. Using these identified seminar users, we build a classification model using a variety of content-based and network features.

- As a case study, we use the classifier to identify and study pro- and anti-Sisi seminar users. Specifically, we examine $(i)$ how much influence such users have on normal users, $(i i)$ whether seminar users are colluding, and (iii) whether there is any interaction between seminar users from opposing sides.

The remainder of this paper is organized as follows. First, we discuss some related work. Then, we present our experiments in finding seminar users. This is followed by a case study of pro- and anti-Sisi seminar users. Finally, we conclude with a general discussion and possible directions for future work.

\section{Related Work}

The behavior of seminar users is closely related to other manipulative and potentially disruptive practices in social media, including discussion trolling, political astroturfing, sockpuppeting, and use of Internet water army, which we outline below. We further discuss some recent work that analyzes political speech in Arabic.

The promise of social media to democratize content creation [27] has also been accompanied by many malicious attempts to spread misleading information over this new medium. News community forums in particular saw the rise and proliferation of fake news [25], aggressiveness [40], and trolling [13]. The latter often is understood to concern malicious online behavior that is intended to disrupt interactions, to aggravate interacting partners, and to lure them into fruitless argumentation in order to disrupt online interactions and communication [11]. Thus, Twitter has taken measures to suspend users who are recognized to be malicious [60]. Nevertheless, more sophisticated malicious profiles such as opinion manipulation trolls (paid [38] or just perceived [37]), sockpuppets [7, 28,31,35], and Internet water army [11] are still barely detectable.

Sockpuppets are people who assume a false identity in an Internet community and then speak to/about themselves while pretending to be another person. The term has also been used to refer to opinion manipulation, e.g., in Wikipedia [53]. Sockpuppets have been identified using authorship-identification techniques and link analysis [7]. It has been also shown that sockpuppets differ from ordinary users in their posting behavior, linguistic traits, and social network structure; moreover, sockpuppets tend to start fewer discussions, write shorter posts, use more personal pronouns such as "I", and have more clustered ego-networks [28]. Furthermore, gangs of sockpuppets controlled by a single person can be identified by the similarity of sentiment orientation toward topics based on their posted comments [31]. Unlike sockpuppets, seminar users do not try to hide their identity. 
Internet water army is a literal translation of the Chinese term wangluo shuijun, which is a metaphor for a large number of people who are well organized to flood the Internet with purposeful comments and articles. Internet water army is allegedly used in China by the government (known also as 50 Cent Party as people involved in the campaign were allegedly paid about 50 cents per post) as well as by private organizations. Chen \& al. [11] used semantic analysis and some non-semantic features such as percentage of replies among the posts, average time between posts, number of active days, and number of news posts a user has commented on. Unlike Internet water army users, seminar users could act alone and are consistent in their support for a given entity over a long period of time.

Trolling behavior is present in all kinds of online media: online magazines [6], social networking sites [13], online computer games [56], online encyclopedia [51], government e-petition pages [58], online newspapers [47], etc. Trolling can be dangerous, as it can increase the risk of suicidal behavior and self-harm amongst the users it is targeted at [52]. Troll detection has been addressed using semantic analysis [9], domain-adapted sentiment analysis [49], various lexico-syntactic features about user writing style and structure [12], as well as graph-based approaches over signed social networks [45]. There have been also studies on general troll behavior [8] and cyber-bullying [48], as well as on linking fake troll profiles to real users [21]. The term troll is often used in popular culture to designate users who engage in opinion manipulation in social media and Web forums; it has been also shown that users who have been called a troll by several different people have common characteristics with paid opinion manipulators [39]. Although similar in their ability to influence discourse, seminar users do not employ as disruptive language as trolls. Instead, they push a consistent political message, which is more closely aligned to astroturfing, and more precisely a kind of an Internet water army [11].

Astroturfing. Named after a brand of fake plastic grass, astroturfing is an effort to simulate a fake political grassroots movement. Recent preoccupation with it has been motivated by strong interest from political science, and research methods driven by the presence of massive streams of microblogging data, largely centering around the Twitter accounts involved. Since Ratkiewicz et al. proposed a system to detect astroturfing called Truthy [46], studies have shown that people can be poor judges of a tweet's credibility [41]. Subsequently, tools to automatically assess the credibility of tweets and their origins spanned both political news [10] and disaster response [23], as well as for the related tasks of stance classification [62] and contradiction detection in rumors [29]. Finally, Lukasik et al. [32] and Ma et al. [33] used temporal patterns to detect rumors, and Zubiaga et al. [63] focused on conversational threads.

Identification of malicious accounts in social networks reaches beyond fake political grassroots movements, and includes detecting spam accounts $[4,36]$, fake accounts [14], compromised accounts and phishing accounts [2]. Fake profile detection has also been studied in the context of cyber-bullying [22]. 
Web spam detection is another related problem, which has been addressed as a text classification problem [50], e.g., using spam keyword spotting [17], lexical affinity of arbitrary words to spam content [26], frequency of punctuation and word co-occurrence [30]. Yet another related problem is that of detecting racist/radicalized posts, e.g., on the Tumblr micro-blogging website [3].

Predicting online extremism is a topic, which involves fighting with a special type of malicious users, very similar to the seminar users group. The goal in [20] is to find Twitter accounts used by ISIS members and to report them to Twitter. After the manual annotation of some ISIS supporters, the authors used a classifier (based on logistic regression and random forests) to predict whether a regular user would retweet a message posted by an ISIS account [20].

Social bot detection involves detecting accounts that are programmatically controlled to produce content and to interact with other users. Social bots may spread messages, ads, or propaganda while trying to mimic human behavior [57]. Varol et al. [57] detected bots using profile, friends, network, temporal, content, and sentiment features. For detecting seminar users, we use features such as lexical diversity and sentiment, which overlap with their features. For social bots in the Arab world, Morstatter et al. [42] looked for keywords that relate to the Arab Spring in Libya. They used several features such as retweet and URL sharing frequencies, tweet length, time between tweets, and topic distribution. Abokhodair et al. [1] looked at the behavior of a social botnet related to the ongoing civil war in Syria and the propensity of the botnet in influencing the mainstream discourse.

Unlike the work above, here we focus on a particular kind of malicious users, i.e., seminar users, which show dedicated support to a particular political entity, acting alone or as part of an organized group.

\section{Identifying Seminar Users}

\section{Data}

We collected Arabic tweets between December 1, 2015 and January 25, 2016. This period is interesting as it constitutes the days leading up to the fifth anniversary of the January 25, 2011 uprising in Egypt, which is part of the Arab Spring and led to the ouster of Hosny Mubarak from power. Though the dates are unlikely to affect the first part of the work concerning the automated detection of seminar users, it would likely make subsequent analysis of specific pro- and anti-Sisi seminar users more interesting. We collected the tweets using the Twitter4j Java interface for the Twitter streaming API, where we searched using the query "lang:ar" to get Arabic tweets. Eventually, we accumulated 417 million tweets with an average of 7.4 million tweets per day over 56 days. We manually labeled 150 users as seminar users vs. non-seminar users. To label them, we picked users who discussed potentially politically polarizing issues about Egypt, United Arab Emirates (UAE), Yemen, and the Kingdom of Saudi Arabia (KSA). 
Specifically, we randomly picked 2,000 users (500 for each country) who mentioned General Sisi of Egypt, Mohamed bin Zayed (vice president of UAE), Yemen, and Saudi Arabia in their tweets. Next, an annotator who is privy to the politics of the Middle East manually examined the tweets of the 2,000 users in random order until he tagged 150 users as fitting our definition of seminar users or not. In the interest of data cleanliness, the annotator labeled users who were clearly seminar users or were clearly not, and he excluded borderline (ambiguous) users where he could not make a clear-cut decision. The annotator was asked to keep the dataset balanced with a comparable number of seminar and non-seminar users. Of the 150 users, 71 were seminar users and 79 were not. To ensure the quality of the annotation, another annotator was asked to independently tag a subset of the tagged users containing 35 users and both annotators agreed on 32 users (91.4\% agreement, with a Cohen's Kappa of 0.83 ).

Tagging users as seminar users is quite different from tagging them as bots. To demonstrate the difference, we automatically tagged all seminar users from our dataset using BotOrNot, ${ }^{6}$ which gives a score between 0 and 100 to each user, with 100 signifying the most extreme bot-like behavior [18]. Figure 1 shows the scores for the seminar users (after excluding 16 accounts that were deleted, protected, or suspended). As the figure shows, BotOrNot reckoned that most of the accounts were not bot-like, with only one user getting the highest score of 61 for any user in our dataset. This may indicate that the accounts are being managed by humans. However, this does not preclude the possibility that some user might manage more than one account.

\section{Detecting Seminar Users}

Methodology Given our set of 150 users that were tagged as seminar users or not, we trained an SVM classifier with a radial basis function kernel ${ }^{7}$ using a variety of features:

- (Interaction features) The relative use of tweet interaction elements. Specifically, the percentage of tweets containing retweets, URLs, hashtags, mentions, and embedded images. The rationale for these features is that seminar users may engage heavily in the promotion of hashtags or memes. ( 5 features)

- (Diversity features) The diversity of content, where seminar users may concentrate on specific topics and use a smaller vocabulary. We measured

- the percentage of tweets containing the top $n$ retweeted accounts or mentioned accounts, where $n$ was set to $1,3,10$, and 20 (8 features);

- the percentage of tweets containing the top $n$ used hashtags for each user, where $n$ was set to 5 and 15 ( 2 features);

- the percentage of tweets containing the top $n$ used words (excluding stopwords) for each user, where $n$ was set to 10,30 , and 50 . We performed basic Arabic letter normalization [16] and English case folding. (3 features)

\footnotetext{
${ }^{6}$ http://truthy.indiana.edu/botornot/

7 We used the SVM ${ }^{\text {Light }}$ implementation available from http://svmlight.joachims.org/
} 


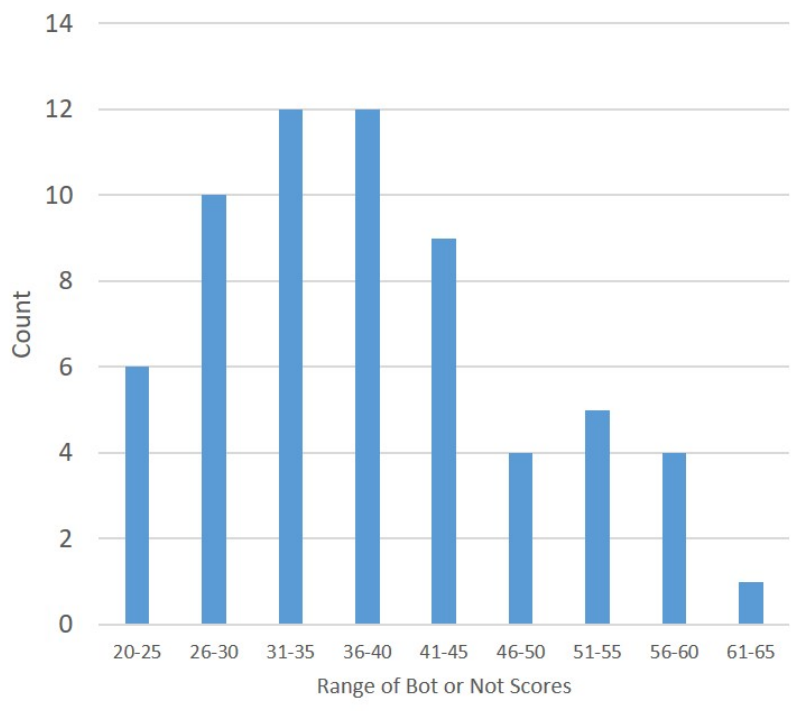

Fig. 1. Distribution of BotOrNot scores for seminar users in our dataset

- (Style features) The style of language, where seminar users are more likely to praise the party they support and malign opponents. We used the following:

- The percentage of tweets containing sentiment words. We used the sentiment lexicon of 3,982 words described in [44]. (1 feature)

- The percentage of tweets containing vulgar or combative words. We used a word list containing Arabic vulgar and obscene words containing 3,840 words and phrases such as "son of a dog" and "filthy" [43]. (1 feature)

We avoided using user similarity features, as such features are computationally expensive. The advantage of our proposed features is that each user can be classified in isolation, without considering the other users, which makes classification computationally efficient. Also, since many users subscribe to services that post mostly religious tweets on their behalf, we assembled a list of the most popular such services and filtered out all tweets that they generate. The services we excluded are du3a.org, ghared.com, 7asnat.com, mezani.net, d3waapp.org, zad-muslim.com, and rtw8.com. We also excluded tweets containing the hashtags \#quran and \#hadith, or that were retweeted from specific accounts that specifically indicate religious content.

Evaluation Due to the limited number of examples in our dataset, we used leave-one-out cross-validation for training and testing, where we held each of the users in our set out for testing and trained on the remaining users. We experimented with using the Interaction, Diversity, and Style features separately and in combination. 
Table 1 reports the classification results using precision $(\mathrm{P})$, recall $(\mathrm{R})$, and F1-measure (F1). We can see that the interaction features are the most important ones. While the combination of all feature types yields the best results, using a combination of interaction and diversity features only, which are languageindependent, slightly degrades the performance. This gives us hope that the classifier may work effectively for users in other regions without the need for language-specific resources. Overall, the results show that we can identify seminar users with relatively high precision and recall.

\begin{tabular}{r|c|c|c|c|c|c|c} 
& \multicolumn{3}{|c|}{ Seminar } & \multicolumn{3}{c|}{ Normal } & Macro \\
Features & $\mathrm{P}$ & $\mathrm{R}$ & $\mathrm{F} 1$ & $\mathrm{P}$ & $\mathrm{R}$ & $\mathrm{F} 1$ & $\mathrm{~F} 1$ \\
\hline Interaction & 77.2 & 62.0 & 68.8 & 71.0 & 83.5 & 76.7 & 72.8 \\
Diversity & 73.2 & 57.7 & 64.6 & 68.1 & 81.0 & 74.0 & 69.3 \\
Style & 0.0 & 0.0 & 0.0 & 52.7 & $\mathbf{1 0 0 . 0}$ & 69.0 & 34.5 \\
Interaction + Diversity & 83.9 & 73.2 & 78.2 & 78.4 & 87.3 & 82.6 & 80.4 \\
\hline All & $\mathbf{8 4 . 4}$ & $\mathbf{7 6 . 1}$ & $\mathbf{8 0 . 0}$ & $\mathbf{8 0 . 2}$ & 87.2 & $\mathbf{8 3 . 6}$ & $\mathbf{8 0 . 9}$
\end{tabular}

Table 1. Classification results for seminar users and normal (non-seminar) users including precision $(\mathrm{P})$, recall $(\mathrm{R})$, F1-measure (F1), and Macro F1-measure.

\section{Pro- and Anti-Sisi Seminar Users: Case Study}

\section{Data}

For the second part of the study, we focus on the behavior of pro- and anti-Sisi seminar users. On July 3, 2013, General Sisi used the military to overthrow the democratically elected government that transpired after January 25, 2011 Egyptian uprising. He was named president a year later. Sisi is a divisive figure in Egyptian politics. We identified 9,506 users who mentioned Sisi ten or more times in our aforementioned dataset containing tweets that are collected between December 1, 2015 and January 25, 2016. To label users as pro- or anti-Sisi, we used label propagation. We manually tagged an initial set of 100 users. Then, we automatically tagged all the tweets mentioning Sisi for those 100 users using their user tags. For example, all tweets mentioning Sisi and posted by pro-Sisi users would be tagged as pro-Sisi. It is not unreasonable to do so as people's opinions generally remain stable over extended periods of time. In particular, we assumed that the overwhelming majority of users would not change their opinion over the span of 56 days. We further assumed that unlabeled users who retweeted consistently pro-Sisi tweets should be pro-Sisi, and likewise those who consistently retweeted anti-Sisi tweets should be anti-Sisi. Thus, we were able to tag 2,743 more users. We repeated this process for three iterations, which ultimately yielded 7,427 tagged users. To check the reliability of label propagation on our data, we took a random sample of 100 users and we labeled them for stance manually and also automatically, obtaining the same label for 99 users. 
We applied our seminar user detector on the 7,427 users that we identified as proand anti-Sisi. As a result, we identified 1,839 users as seminar users, including 492 who were pro-Sisi and 1,347 who were anti-Sisi. In order to verify the efficacy of the classifier on the new data, we randomly sampled 50 users from the set of 7,427 users, and we asked our annotator to manually and independently label them as seminar users or not. The annotator labeled 20 users as seminar users, 29 as normal users, and 1 as a spammer. Our classifier labeled 14 (out of the 50) users as seminar users, of which 11 were in fact seminar users (Precision $=0.78$; Recall $=0.55)$. Unlike the training data, not all users were clearly discernible as seminar users or not. The annotator labeled all ambiguous users (8 users) using his best guess. This likely contributed to lower results over the sample compared to the aforementioned classification results.

Table 2 summarizes the number of users for each group and other basic statistics about them. The summary shows that the ratio of seminar users to normal users is 1:1.7 and 1:3.5 for pro- and anti-Sisi users, respectively. It is noteworthy that pro-Sisi seminar users authored more tweets than pro-Sisi nonseminar users. Conversely, normal anti-Sisi users produced more than twice as many tweets as anti-Sisi seminar users. Seminar users produced on average 60\% more tweets per user, and they tweeted more on average about Sisi.

\begin{tabular}{l|r|r|r|r} 
& \multicolumn{2}{|c|}{ Seminar Users } & \multicolumn{2}{c}{ Normal Users } \\
\hline Stance & Pro-Sisi & Anti-Sisi & Pro-Sisi & Anti-Sisi \\
\hline Users & 492 & 1,347 & 846 & 4,748 \\
Tweets & 860,085 & $4,200,619$ & 847,383 & $9,057,758$ \\
Avg tweets/user & 1,748 & 3,118 & 1,001 & 1,908 \\
Tweets mentioning Sisi & 22,932 & 65,478 & 34,026 & 146,762 \\
Avg tweets mentioning Sisi/user & 46.6 & 48.6 & 40.2 & 30.9
\end{tabular}

Table 2. Stats of pro- and anti-Sisi users and tweets.

\section{Characteristics of Pro- and Anti-Sisi Seminar Users}

Figure 2 shows the top- 5 self-declared user locations producing the highest number of pro- and anti-Sisi tweets. We can see that most users did not declare their location. Moreover, even though there were far fewer pro-Sisi users from UAE compared to Egypt, they produced five times as many tweets. Similarly for anti-Sisi users, there were nearly six times as many users from Egypt than from KSA, but the number of tweets from Egypt were only 19\% more than those from KSA. This may indicate that Egyptian seminar users are less active than non-Egyptian ones. Table 3 lists the top retweeted accounts by both groups. As the table shows, for both groups, the majority of these accounts were not from Egypt, and UAE and KSA users account for most pro-Sisi seminar users. 

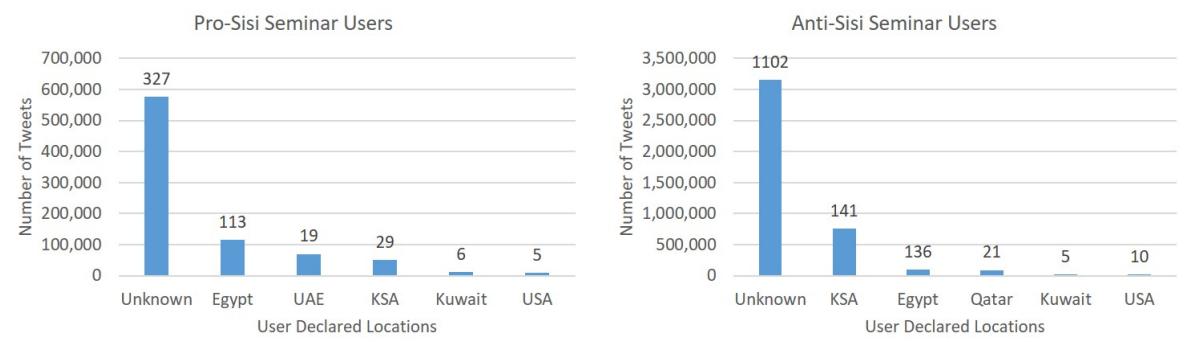

Fig. 2. Top-5 self-declared user locations of pro- and anti-Sisi seminar users. Values at the top of the bars indicate the number of users.

\begin{tabular}{r|l|r|r|r|r}
\multicolumn{2}{c|}{ Pro-Sisi } & \multicolumn{2}{c}{ Anti-Sisi } \\
\hline User & Description & Count & User & Description & Count \\
\hline Forsan_UAE & UAE user & 33,229 & ALAMAWI & Syrian pro-Arab Spring media & 58,203 \\
HenryKesnger & Unknown & 10,452 & almokhtsar & News aggregator & 29,968 \\
fdeet_alnssr & KSA & 7,817 & raawnq & suspended & 26,117 \\
meshaluk & KSA user in London & 5,840 & EHSANFAQEH & Jordanian author & 23,005 \\
alkhaldi_ksa & suspended & 4,578 & Wesal_TV & KSA (anti-Iran media) & 19,767 \\
Dhahi_Khalfan & UAE user & 4,258 & S7K00 & suspended & 19,403 \\
bestwalid92 & Egypt & 3,778 & AJArabic & Aljazeera (media) & 18,212 \\
amirelghareb & Egypt user & 3,696 & kasimf & Aljazeera presenter & 15,492 \\
a_e5552000 & suspended & 3,627 & AboShla5Libraly & KSA user & 14,389 \\
shereen_hussen & Egypt user & 3,502 & YZaatreh & Palestinian author & 13,639
\end{tabular}

Table 3. Top retweeted accounts for pro- and anti-Sisi seminar users.

Seminar users typically engage in campaigns to promote specific messages typically with associated hashtags. We identified such campaigns by identifying hashtags that combine both high volume and high standard deviation $(\sigma)$ from day to day. Thus, we scored hashtags by combining volume and standard deviation:

$$
\text { score }=\frac{\sigma}{\sum \text { daily_counts }}
$$

We only considered hashtags that were used more than 100 and 300 times for pro- and anti-Sisi groups, respectively, and we ranked them using scoring formula (1). We picked different thresholds for the two groups in order to account for the difference in volume. Figure 3 shows the top- 15 hashtag campaigns for both groups. We can see that the campaigns typically lasted for one or two days and then died out. The pro-Sisi group was involved in continuous campaigns, while the anti-Sisi group campaigns started at the end of December as the anniversary of the 2011 Egyptian uprising drew closer. For the pro-Sisi group, 3 out of 15 of the top hashtags were related to Egypt and 7 were related to UAE. In contrast, 14 out of 15 hashtags were related to Egypt in the anti-Sisi group. 

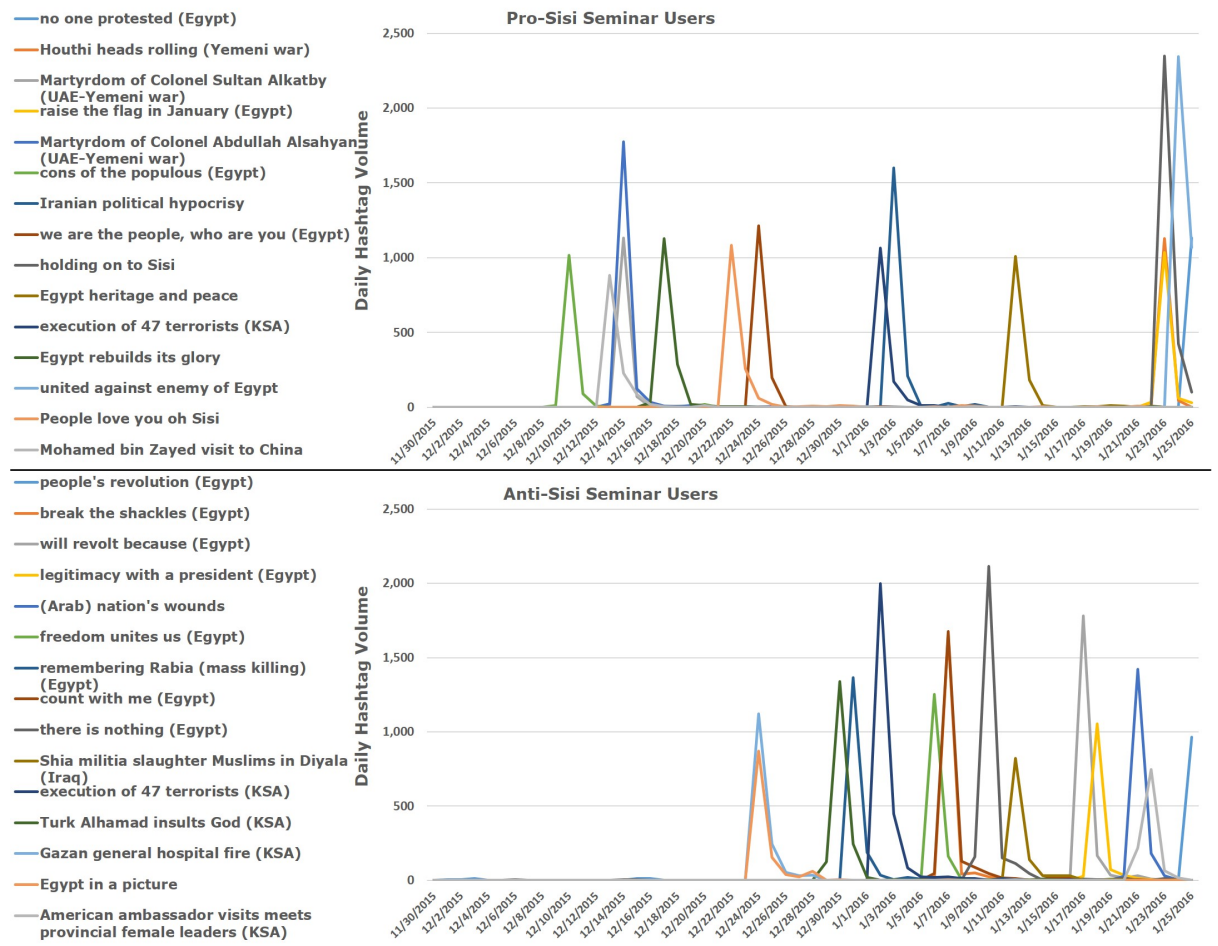

Fig. 3. Top hashtag campaigns for pro- and anti-Sisi seminar users.

\section{Effectiveness, Cohesiveness, and Interactions between Seminar Users}

We set out to answer three research questions aimed to assess the effectiveness and the cohesiveness of seminar users, and the extent to which they interact with each other. Our first question is the following: how successful are seminar users in penetrating the mainstream? To answer this question, we considered the 5,618 pro- and anti-Sisi users that our classifier deemed as non-seminar users.

We considered the top-100 most used hashtags with a score greater than 0.02 , using formula (1). Then, we compared the top-100 hashtags for the proand anti-Sisi seminar groups to the top hashtags for non-seminar users, focusing on the following statistics: $(i)$ percentage of hashtags that made the list from non-seminar users, $(i i)$ average rank of hashtags in the list, and ( $i i i)$ volume magnification factor, which is the volume of hashtags from non-seminar users divided by the volume generated by seminar users. Table 4 reports on these three statistics for the pro- and anti-Sisi seminar users. We can see that antiSisi seminar users were more successful than pro-Sisi ones in penetrating the mainstream with $29 \%$ (compared to 10\%) of their top-100 hashtags appearing in the top hashtags for non-seminar users with an average rank of 22.6 (vs. 46.1). However, volume magnification for pro-Sisi users is much higher (9.0 vs. 1.6). 
Three caveats need to be taken into account when reading these results: $(i)$ antiSisi seminar users outnumber pro-Sisi ones by a factor 2.7 to 1 , (ii) pro-Sisi seminar users do not concentrate on Egypt-related issues, and ( iii) seminar users may adopt an existing hashtag, instead of introducing a new one.

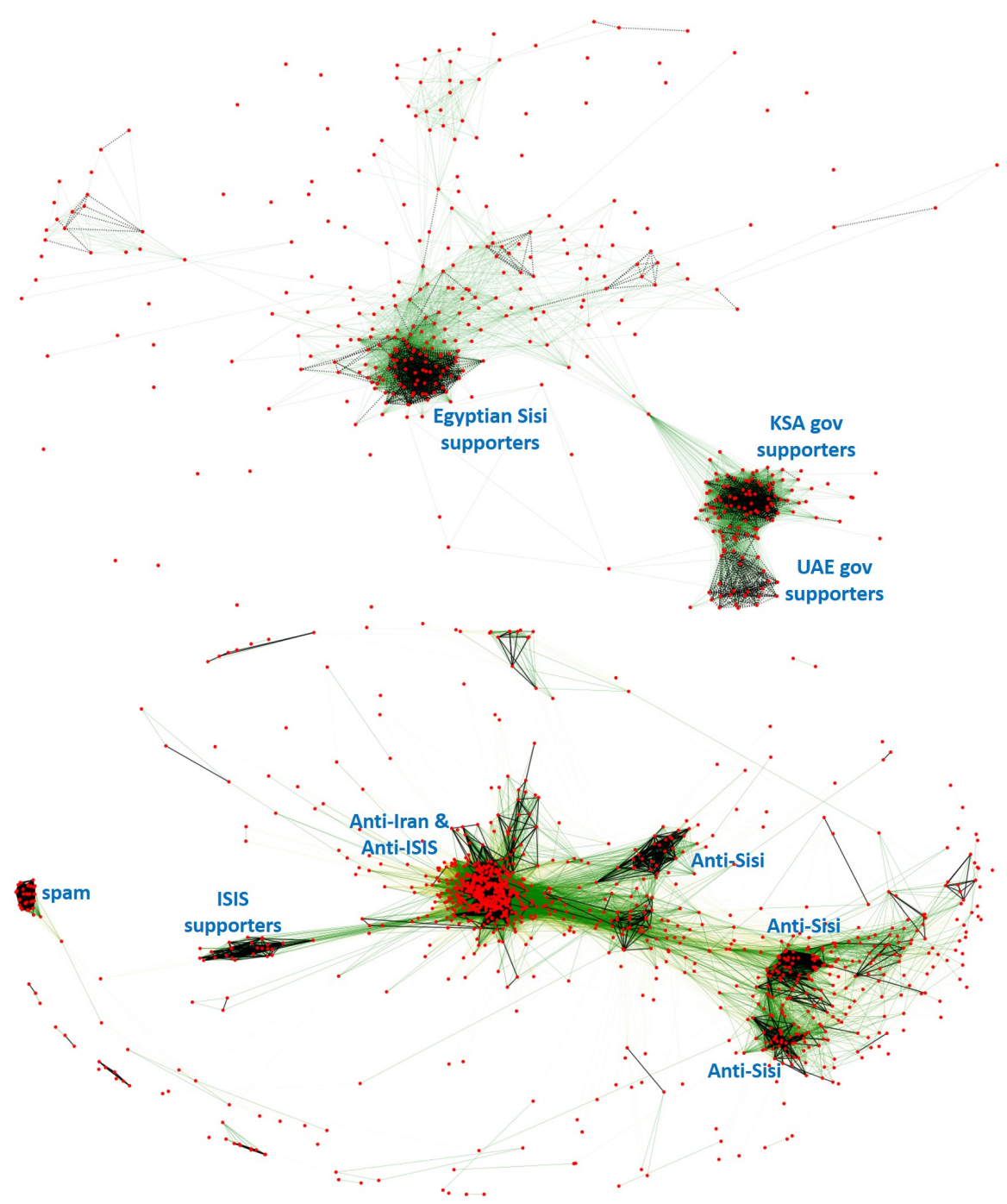

Fig. 4. Pro- and anti-Sisi user network graph (top and bottom, respectively). We indicate cosine similarity greater than 0.8 as black edges, between 0.6 and 0.8 as green edges, and less than 0.6 as light yellow edges. We further show text labels for the main strongly-connected cliques. 
Our second research question we study is the following: how similar are seminar users in each camp? To answer this question, we computed the cosine similarity between pro- and anti-Sisi seminar users using the frequency of the hashtags that they used as features. Next, we drew the network of users shown in Figure 4 using NetworkX, ${ }^{8}$ which uses Fruchterman-Reingold force-directed algorithm to space out the nodes. As the network graphs show, the seminar users are not homogeneous, but rather they are groups of strongly-connected cliques. We labeled the major cliques in the graphs based on sample users from each clique. We can see that pro-Sisi seminar users are composed of three major cliques from Egypt, KSA, and UAE. Given the apparent skew toward UAE-related topics, it seems that the pro-UAE group is most productive in terms of volume. For the anti-Sisi seminar users, there is a large anti-Iran group, which is also pro-Syrian revolutionaries, there are three different Egyptian anti-Sisi groups, one pro-Islamic State (ISIS) group, and one group of spammers. All of these groups are supportive of the Arab Spring uprisings in one way or another. Prior work has discussed the relationship between support for ISIS and the Arab Spring [34].

Our third research question is the following: how much do the two groups of seminar users interact? To answer this question, we measured whether the two camps used the same hashtags. In essence, we wanted to detect hashtag hijacking. We found that the two camps shared only nine of the top 100 most frequent hashtags, and the rest were used exclusive by one of them. This included seven shared hashtags of broad interest such as \#KSACutsDiplomaticTies WithIran and \#DubaiFire. The other two hashtags were \#PeopleWantToOverthrowRegime (used $96 \%$ of the time by the anti-Sisi camp) and \#KnowThePerpetrator (used $65 \%$ of the time by the pro-Sisi camp). The results suggest that both pro- and anti-Sisi seminar users operate fairly independently and with limited interaction.

\begin{tabular}{r|c|c|c} 
& \% of appearance & Avg. Rank & Vol. magnification \\
\hline pro-Sisi & $10 \%$ & 46.1 & $9.0 \mathrm{x}$ \\
anti-Sisi & $29 \%$ & 22.6 & $1.6 \mathrm{x}$
\end{tabular}

Table 4. Penetration of pro- and anti-Sisi seminar users into the mainstream.

\section{Discussion and Conclusions}

We have introduced the so-called "seminar users", as users who engage in political propaganda. The existence of such users can distort social media analysis and can be socially and politically troubling. Thus, we have presented a robust method for automatically detecting such users by looking at their interactions, their tweet content diversity, and their tendency to use sentiment-bearing and offensive words. Note that we did not use user similarity features as mining the user graph of interactions is computationally expensive.

\footnotetext{
${ }^{8}$ http://networkx.readthedocs.io/en/networkx-1.10/
} 
Our seminar vs. regular user classifier achieved precision of $84.4 \%$ and recall of $76.1 \%$ on our dataset, which is a very strong result. We have further demonstrated that seminar users are quite different from bots. Complementary to recent research that has shown that it is possible to distinguish automatically real human Twitter accounts from bots [55], our work points to a need for detecting networks of colluding Twitter users, beyond the detection of ephemeral bot accounts.

We further applied our classifier on Twitter users who discuss a politically controversial topic, namely attitude toward General Sisi of Egypt. Our classifier labeled $25 \%$ of these users as seminar users, but we suspect that the actual percentage should be even higher. Moreover, our analysis shows that seminar users produce more tweets on average compared to normal users, e.g., more than half of the pro-Sisi and about a third of the anti-Sisi tweets in our dataset were posted by seminar users. We further found that seminar users are often successful in affecting the mainstream discourse, which is evident by the hashtags that they popularize. The prevalence of seminar users in the Arabic Twitter-sphere and the large volume of tweets that they produce complicate Twitter-based studies, particularly for controversial or political issues. The success of seminar users in affecting the mainstream has the potential of promoting human rights abuses, or making such abuses sound like a normal thing. Thus, the ability to detect such users can help analysis in two major ways, namely $(i)$ filtering out such users can help focus analysis on normal users, and (ii) focusing exclusively on seminar users can help elucidate what political actors are promoting aside from what they say officially.

We have shown that seminar users with similar stances on specific issues may actually belong to wildly different or even opposing groups of Twitter users, and that they may even come from different countries altogether. In particular, for anti-Sisi seminar users, we can clearly see a group that supports ISIS and another larger group that opposes ISIS. In the example of pro-Sisi seminar users, we have seen such users from UAE and KSA, who work across borders to support Sisi and to oppose his adversaries. Moreover, we have shown that groups of seminar users may or may not engage with the hashtags of opposing groups, e.g., in the form of hashtag hijacking. Overall, in our case study, we have found very limited evidence for this kind of cross-group engagement. We suspect that the seminar users phenomenon is not unique to the Arab region, but exists in different regions of the world with varying potency. We plan to test whether our seminar vs. normal users classifier can generalize to other regions of the world, and whether seminar users behave similarly across regions and whether they share some universal characteristics.

Last but not least, by focusing our study on the political issues in the Arab world, we contribute to a growing literature examining the role of social media in political communication around the world. Although studies about United States are most prominent (e.g., those on the Presidential elections [61]), there is also recent work focusing on Latin America [59], China [54], Europe [15], and the Middle East [34]. We hope that the present work would enable further research on the role and use of social media in Arab politics. 


\section{References}

1. Abokhodair, N., Yoo, D., McDonald, D.W.: Dissecting a social botnet: growth, content and influence in Twitter. In: Proceedings of the 18th ACM Conference on Computer Supported Cooperative Work \& Social Computing. pp. 839-851. CSCW '15, Vancouver, British Columbia, Canada (2015)

2. Adewole, K.S., Anuar, N.B., Kamsin, A., Varathan, K.D., Razak, S.A.: Malicious accounts: Dark of the social networks. Journal of Network and Computer Applications 79, 41-67 (2017)

3. Agarwal, S., Sureka, A.: Characterizing linguistic attributes for automatic classification of intent based racist/radicalized posts on Tumblr micro-blogging website. arXiv preprint arXiv:1701.04931 (2017)

4. Almaatouq, A., Shmueli, E., Nouh, M., Alabdulkareem, A., Singh, V.K., Alsaleh, M., Alarifi, A., Alfaris, A., et al.: If it looks like a spammer and behaves like a spammer, it must be a spammer: Analysis and detection of microblogging spam accounts. International Journal of Information Security 15(5), 475-491 (2016)

5. Benevenuto, F., Magno, G., Rodrigues, T., Almeida, V.: Detecting spammers on Twitter. In: Proceedings of the Conference on Collaboration, Electronic Messaging, Anti-abuse and Spam. CEAS '10, vol. 6, p. 12. Redmond, Washington, USA (2010)

6. Binns, A.: DON'T FEED THE TROLLS! Managing troublemakers in magazines online communities. Journalism Practice 6(4), 547-562 (2012)

7. Bu, Z., Xia, Z., Wang, J.: A sock puppet detection algorithm on virtual spaces. Know.-Based Syst. 37, 366-377 (Jan 2013)

8. Buckels, E.E., Trapnell, P.D., Paulhus, D.L.: Trolls just want to have fun. Personality and Individual Differences 67, 97-102 (2014)

9. Cambria, E., Chandra, P., Sharma, A., Hussain, A.: Do not feel the trolls. In: Proceedings of the 3rd International Workshop on Social Data on the Web. SDoW '10, Shanghai, China (2010)

10. Castillo, C., Mendoza, M., Poblete, B.: Predicting information credibility in timesensitive social media. Internet Research 23(5), 560-588 (2013)

11. Chen, C., Wu, K., Srinivasan, V., Zhang, X.: Battling the Internet Water Army: detection of hidden paid posters. In: Proceedings of the 2013 IEEE/ACM International Conference on Advances in Social Networks Analysis and Mining. pp. 116-120. ASONAM '13, Niagara, Ontario, Canada (2013)

12. Chen, Y., Zhou, Y., Zhu, S., Xu, H.: Detecting offensive language in social media to protect adolescent online safety. In: Proceedings of the 2012 International Conference on Privacy, Security, Risk and Trust and of the 2012 International Conference on Social Computing. pp. 71-80. PASSAT/SocialCom '12, Amsterdam, Netherlands (2012)

13. Cole, K.K.: "It's like she's eager to be verbally abused": Twitter, trolls, and (en) gendering disciplinary rhetoric. Feminist Media Studies 15(2), 356-358 (2015)

14. Cresci, S., Di Pietro, R., Petrocchi, M., Spognardi, A., Tesconi, M.: Fame for sale: efficient detection of fake Twitter followers. Decision Support Systems 80, 56-71 (2015)

15. Cross, J.P., Greene, D., Belford, M.: Tweeting Europe: A text-analytic approach to unveiling the content of political actors' Twitter activities in the European parliament. In: Proceedings of 6th Annual General Conference of the European Political Science Association. EPSA'16, Brussels, Belgium (2016)

16. Darwish, K., Magdy, W., Mourad, A.: Language processing for Arabic microblog retrieval. In: Proceedings of the 21st ACM International Conference on Information and Knowledge Management. pp. 2427-2430. CIKM '12, Maui, Hawaii, USA (2012) 
17. Dave, K., Lawrence, S., Pennock, D.M.: Mining the peanut gallery: Opinion extraction and semantic classification of product reviews. In: Proceedings of the 12th International World Wide Web conference. pp. 519-528. WWW '03, Budapest, Hungary (2003)

18. Davis, C.A., Varol, O., Ferrara, E., Flammini, A., Menczer, F.: BotOrNot: A system to evaluate social bots. In: Proceedings of the 25th International Conference Companion on World Wide Web. pp. 273-274. WWW '16, Montréal, Québec, Canada (2016)

19. Ferrara, E., Varol, O., Davis, C., Menczer, F., Flammini, A.: The rise of social bots. Commun. ACM 59(7), 96-104 (Jun 2016)

20. Ferrara, E., Wang, W.Q., Varol, O., Flammini, A., Galstyan, A.: Predicting online extremism, content adopters, and interaction reciprocity. In: Proceedings of 8th International Conference. pp. 22-39. SocInfo '16, Bellevue, Washington, USA (2016)

21. Galán-García, P., De La Puerta, J.G., Gómez, C.L., Santos, I., Bringas, P.G.: Supervised machine learning for the detection of troll profiles in Twitter social network: Application to a real case of cyberbullying. Logic Journal of IGPL pp. 42-53 (2015)

22. Galán-García, P., de la Puerta, J.G., Gómez, C.L., Santos, I., Bringas, P.G.: Supervised machine learning for the detection of troll profiles in Twitter social network: Application to a real case of cyberbullying. In: Proceedings of the International Joint Conference SOCO'13-CISIS'13-ICEUTE'13, pp. 419-428. Advances in Intelligent Systems and Computing (2014)

23. Gupta, A., Lamba, H., Kumaraguru, P., Joshi, A.: Faking Sandy: characterizing and identifying fake images on Twitter during hurricane Sandy. In: Proceedings of the 22nd International Conference on World Wide Web. pp. 729-736. WWW '13 (2013)

24. Hardaker, C.: Trolling in asynchronous computer-mediated communication: from user discussions to theoretical concepts. Journal of Politeness Research 6(2), 215-242 (2010)

25. Hardalov, M., Koychev, I., Nakov, P.: In search of credible news. In: Proceedings of the 17th International Conference on Artificial Intelligence: Methodology, Systems, and Applications. pp. 172-180. AIMSA '16, Varna, Bulgaria (2016)

26. $\mathrm{Hu}, \mathrm{M}$., Liu, B.: Mining and summarizing customer reviews. In: Proceedings of the 10th ACM SIGKDD International Conference on Knowledge Discovery and Data Mining. pp. 168-177. KDD '04, Seattle, Washington, USA (2004)

27. Kaplan, A.M., Haenlein, M.: Users of the world, unite! The challenges and opportunities of social media. Business horizons 53(1), 59-68 (2010)

28. Kumar, S., Cheng, J., Leskovec, J., Subrahmanian, V.: An army of me: sockpuppets in online discussion communities. In: Proceedings of the 26th International Conference on World Wide Web. pp. 857-866. WWW '17, Perth, Australia (2017)

29. Lendvai, P., Reichel, U.D.: Contradiction detection for rumorous claims. arXiv preprint arXiv:1611.02588 (2016)

30. Li, W., Zhong, N., Liu, C.: Combining multiple email filters based on multivariate statistical analysis. In: Proceedings of the 16th International Conference on Foundations of Intelligent Systems. pp. 729-738. ISMIS '06, Bari, Italy (2006)

31. Liu, D., Wu, Q., Han, W., Zhou, B.: Sockpuppet gang detection on social media sites. Front. Comput. Sci. 10(1), 124-135 (Feb 2016)

32. Lukasik, M., Cohn, T., Bontcheva, K.: Point process modelling of rumour dynamics in social media. In: Proceedings of the 53rd Annual Meeting of the Association for Computational Linguistics and the 7th International Joint Conference on Natural Language Processing. pp. 518-523. Beijing, China (2015) 
33. Ma, J., Gao, W., Wei, Z., Lu, Y., Wong, K.F.: Detect rumors using time series of social context information on microblogging websites. In: Proceedings of the 24th ACM International on Conference on Information and Knowledge Management. pp. 1751-1754. CIKM '15, Melbourne, Australia (2015)

34. Magdy, W., Darwish, K., Weber, I.: \#FailedRevolutions: Using Twitter to study the antecedents of ISIS support. First Monday 21(2) (2016)

35. Maity, S.K., Chakraborty, A., Goyal, P., Mukherjee, A.: Detection of sockpuppets in social media. In: Proceedings of the 2017 ACM Conference on Computer Supported Cooperative Work and Social Computing. pp. 243-246. CSCW '17, Portland, Oregon, USA (2017)

36. Mccord, M., Chuah, M.: Spam detection on Twitter using traditional classifiers. In: Proceedings of the International Conference on Autonomic and Trusted Computing. pp. 175-186. ATC '11, Banff, Alberta, Canada (2011)

37. Mihaylov, T., Georgiev, G., Nakov, P.: Finding opinion manipulation trolls in news community forums. In: Proceedings of the Nineteenth Conference on Computational Natural Language Learning. pp. 310-314. CoNLL '15, Beijing, China (2015)

38. Mihaylov, T., Koychev, I., Georgiev, G., Nakov, P.: Exposing paid opinion manipulation trolls. In: Proceedings of the International Conference Recent Advances in Natural Language Processing. pp. 443-450. RANLP '15, Hissar, Bulgaria (2015)

39. Mihaylov, T., Nakov, P.: Hunting for troll comments in news community forums. In: Proceedings of the 54th Annual Meeting of the Association for Computational Linguistics. pp. 399-405. ACL '16, Berlin, Germany (2016)

40. Moore, M.J., Nakano, T., Enomoto, A., Suda, T.: Anonymity and roles associated with aggressive posts in an online forum. Computers in Human Behavior 28(3), 861-867 (2012)

41. Morris, M.R., Counts, S., Roseway, A., Hoff, A., Schwarz, J.: Tweeting is believing?: Understanding microblog credibility perceptions. In: Proceedings of the ACM 2012 Conference on Computer Supported Cooperative Work. pp. 441-450. CSCW '12, Seattle, Washington, USA (2012)

42. Morstatter, F., Wu, L., Nazer, T., Carley, K., Liu, H.: A new approach to bot detection: striking the balance between precision and recall, pp. 533-540. ASONAM'16, San Francisco, California, USA (2016)

43. Mubarak, H., Darwish, K., Magdy, W.: Abusive language detection on Arabic social media. In: Proceedings of the Workshop on Abusive Language Online. ALW '17, Vancouver, British Columbia, Canada (2017)

44. Muhammad, A., Kübler, S., Diab, M.: Samar: a system for subjectivity and sentiment analysis of Arabic social media. In: Proceedings of the 3rd Workshop in Computational Approaches to Subjectivity and Sentiment Analysis. pp. 19-28. WASSA '12, Copenhagen, Denmark (2012)

45. Ortega, F.J., Troyano, J.A., Cruz, F.L., Vallejo, C.G., Enriquez, F.: Propagation of trust and distrust for the detection of trolls in a social network. Computer Networks $56(12), 2884-2895(2012)$

46. Ratkiewicz, J., Conover, M., Meiss, M., Gonçalves, B., Patil, S., Flammini, A., Menczer, F.: Truthy: mapping the spread of astroturf in microblog streams. In: Proceedings of the 20th International Conference on World Wide Web. pp. 249-252. WWW '11, Hyderabad, India (2011)

47. Ruiz, C., Domingo, D., Micó, J.L., Díaz-Noci, J., Meso, K., Masip, P.: Public sphere 2.0? The democratic qualities of citizen debates in online newspapers. The International Journal of Press/Politics 16(4), 463-487 (2011) 
48. Sarna, G., Bhatia, M.: Content based approach to find the credibility of user in social networks: an application of cyberbullying. International Journal Of Machine Learning and Cybernetics pp. 1-13 (2015)

49. Seah, C.W., Chieu, H.L., Chai, K.M.A., Teow, L.N., Yeong, L.W.: Troll detection by domain-adapting sentiment analysis. In: Proceedings of the 18th International Conference on Information Fusion. pp. 792-799. FUSION '15, Washington, DC, USA (2015)

50. Sebastiani, F.: Machine learning in automated text categorization. ACM computing surveys (CSUR) 34(1), 1-47 (2002)

51. Shachaf, P., Hara, N.: Beyond vandalism: Wikipedia trolls. Journal of Information Science 36(3), 357-370 (2010)

52. Slee, P.T., Skrzypiec, G.: School bullying, victimization and pro-social behaviour. In: Well-Being, Positive Peer Relations and Bullying in School Settings, pp. 109-133. Positive Education, Springer (2016)

53. Solorio, T., Hasan, R., Mizan, M.: Sockpuppet detection in Wikipedia: a corpus of real-world deceptive writing for linking identities. In: Proceedings of the Ninth International Conference on Language Resources and Evaluation. pp. 1355-1358. LREC '14, Reykjavik, Iceland (2014)

54. Song, Y., Dai, X.Y., Wang, J.: Not all emotions are created equal: Expressive behavior of the networked public on China's social media site. Computers in Human Behavior 60, 525-533 (2016)

55. Stieglitz, S., Brachten, F., Berthelé, D., Schlaus, M., Venetopoulou, C., Veutgen, D.: Do social bots (still) act different to humans?-Comparing metrics of social bots with those of humans. In: Proceedings of the International Conference on Social Computing and Social Media. pp. 379-395. HCI '17, Vancouver, British Columbia, Canada (2017)

56. Thacker, S., Griffiths, M.D.: An exploratory study of trolling in online video gaming. International Journal of Cyber Behavior, Psychology and Learning (IJCBPL) 2(4), 17-33 (2012)

57. Varol, O., Ferrara, E., Davis, C.A., Menczer, F., Flammini, A.: Online human-bot interactions: detection, estimation, and characterization (2017)

58. Virkar, S.: Trolls just want to have fun: Electronic aggression within the context of e-participation and other online political behaviour in the united kingdom. Int. J. E-Polit. 5(4), 21-51 (2014)

59. Waisbord, S., Amado, A.: Populist communication by digital means: Presidential Twitter in Latin America. Information, Communication \& Society pp. 1-17 (2017)

60. Wei, W., Joseph, K., Liu, H., Carley, K.M.: The fragility of Twitter social networks against suspended users. In: Proceedings of the 2015 IEEE/ACM International Conference on Advances in Social Networks Analysis and Mining 2015. pp. 9-16. ASONAM '15, Paris, France (2015)

61. Wells, C., Shah, D.V., Pevehouse, J.C., Yang, J., Pelled, A., Boehm, F., Lukito, J., Ghosh, S., Schmidt, J.L.: How Trump drove coverage to the nomination: hybrid media campaigning. Political Communication 33(4), 669-676 (2016)

62. Zubiaga, A., Kochkina, E., Liakata, M., Procter, R., Lukasik, M.: Stance classification in rumours as a sequential task exploiting the tree structure of social media conversations. arXiv preprint arXiv:1609.09028 (2016)

63. Zubiaga, A., Liakata, M., Procter, R., Wong Sak Hoi, G., Tolmie, P.: Analysing how people orient to and spread rumours in social media by looking at conversational threads. PLoS ONE 11(3), 1-29 (03 2016) 